\title{
Extremely bright coherent synchrotron radiation production in a diffraction-limited storage ring using an angular dispersion-induced microbunching scheme
}

\author{
Changliang Li® ${ }^{1,2,3}$ Chao Feng, ${ }^{1,2}$ and Bocheng Jiang $\circledast^{1,2, *}$ \\ ${ }^{1}$ Shanghai Institute of Applied Physics, Chinese Academy of Sciences, Shanghai 201800, China \\ ${ }^{2}$ Shanghai Advanced Research Institute, Chinese Academy of Sciences, Shanghai 201204, China \\ ${ }^{3}$ University of the Chinese Academy of Sciences, Beijing 100049, China
}

(Received 17 March 2020; accepted 14 September 2020; published 6 November 2020)

\begin{abstract}
Generation of extremely bright, coherent synchrotron radiation in a short wavelength range is of remarkable interest in the synchrotron light source community. In this paper, a novel technique is adopted to produce coherent radiation, which uses an angular dispersion mechanism to enhance the microbunching of electrons by introducing a slight energy modulation in the long straight section of the storage ring. For this purpose, a lattice design of an extreme low emittance storage ring with $3.5 \mathrm{GeV}$ energy is presented in this paper, which employs a higher-order achromat concept. We show the design results of the multibend achromat lattice with an emittance of $20 \mathrm{pm}$ rad and a circumference of $900 \mathrm{~m}$. Numerical simulation results demonstrate that this angular dispersion-induced microbunching scheme can be used to generate coherent radiation near the soft $\mathrm{x}$-ray region, the repetition rate can reach $10 \mathrm{kHz}$, and the spectral brightness can reach $2.3 \times 10^{24}$ photons s $\mathrm{sm}^{-1} \mathrm{mrad}^{-2}(0.1 \% \text { bandwidth })^{-1}$.
\end{abstract}

DOI: 10.1103/PhysRevAccelBeams.23.110701

\section{INTRODUCTION}

The development of linac-based free-electron laser (FEL) and storage ring-based synchrotron radiation light sources has brought unprecedented revolutionary research tools to the fields of physics, chemistry, life science, and material science and greatly promoted the development of related science [1]. Linac-based FELs not only have the advantages of ultrahigh peak brightness, ultrashort pulse structure, and excellent longitudinal coherence, but also have the ability to continuously adjust the wavelength of the emitted light [2-4]. The emergence of advanced FEL light sources, such as extreme ultraviolet (EUV) and x-ray FELs with high energy and high spatial and temporal resolutions, has provided unprecedented tools for detecting the ultrafast evolution of microscale structures. However, the repetition rate of FELs is limited. While this has been addressed to some extent by the development of high repetition rate electron gun and superconducting linac technology, the cost and technical difficulties associated with these developments are quite substantial. Moreover, FEL light sources can generally supply light to only a

jiangbocheng@zjlab.org.cn

Published by the American Physical Society under the terms of the Creative Commons Attribution 4.0 International license. Further distribution of this work must maintain attribution to the author(s) and the published article's title, journal citation, and DOI. limited number of experimental stations, which further limits the scope of its applications. In contrast, storage ringbased synchrotron light sources have the advantages of wide spectral coverage, high average brightness, high stability, and simultaneous support for multiple users [5]. This technology has undergone three generations of development and evolution since its inception in the 1960s and is currently moving toward a fourth generation aiming at diffraction-limited storage rings with higher brightness and better transverse coherence. Storage ring-based synchrotron radiation light sources have now become a major scientific platform supporting the multidisciplinary development of basic and applied research. However, the electron bunch length in the storage ring is relatively longer than that in the linac, which limits the temporal coherence of the emitted light.

One scheme that has been pursued for addressing the issues confronting linac-based FEL and storage ring-based synchrotron radiation light sources has sought to combine these two technologies and, thereby, support the advantages of both while mitigating their disadvantages $[6,7]$. This scheme involves two primary strategies, where one adopts a low-gain FEL based on an optical resonator, and the other employs coherent harmonic generation FEL (CHG-FEL) technologies. However, resonant cavity FEL is limited by the reflective materials employed, and an output wavelength less than $200 \mathrm{~nm}$ is difficult to achieve. In contrast, CHG-FEL technologies require the use of a conventional laser as a seed and its harmonic conversion to produce 
short-wavelength radiation. An optimal combination of CHG-FEL and storage ring technologies would provide users with an ideal ultrashort pulse, fully coherent light source. However, the number of harmonic conversions of CHG-FEL is limited by the energy spread of the electron beam in the storage ring, which is typically on the order of $10^{-3}$. Moreover, a strong seed laser with a peak power in the range of $1-100 \mathrm{GW}$ is needed to introduce sufficient energy modulation, and the depth of energy modulation must be limited to ensure that the quality of the electron beam is not degraded. Accordingly, these many practical limitations have seriously hampered the development of combined CHG-FEL and storage ring technologies over the past two decades.

Other schemes have been developed to increase the coherency of synchrotron radiation in storage rings. For example, angular-modulated electron beams have been proposed to produce coherent synchrotron radiation in storage rings $[8,9]$. However, no results of proof-ofprinciple experiments have yet been reported. In addition, the femtoslicing method has been proposed to achieve femtosecond radiation pulses in storage ring-based light sources [10-12]. Here, one employs the resonant interaction of an electron bunch with a femtosecond laser beam in a wiggler to modulate the energy of a short section of the bunch. The induced energy modulation is then converted to a transverse displacement using a horizontal or vertical dispersion bump downstream of the wiggler in such a way that the radiation from the femtosecond pulse can be separated from the radiation of the main bunch. The repetition rate obtained using this approach is on the order of $10 \mathrm{kHz}$.

Storage ring-based synchrotron radiation holds the property of a high repetition rate and relatively low peak power. The FEL has a low repetition rate and an extremely high peak power. Among the many applications for highpower coherent radiation sources, some of them do not demand high peak power. Instead, they focus on having a high average power and high repetition rate. To approach high average power radiation, steady-state microbunching (SSMB) has been proposed in Ref. [13]. SSMB is based on an electron storage ring which is much more mature than the energy recovery linac. The latter is also a candidate for high average power radiation provider yet under development [14]. When the microbunch length approaches the radiation wavelength, coherent radiation will be produced, and radiation power will be orders of magnitude higher. Together with the high repetition rate, the high average power radiation will be produced. Beyond the scientific applications, these technologies have some important industrial applications as well, such as EUV lithography. At present, two types of SSMB approaches are under development, which include strong focusing SSMB and reversible SSMB [15-20]. However, both of these are subject to several challenges. With respect to reversible SSMB, the turn-byturn and bunch-by-bunch production of coherent radiation requires not only a novel lattice between the modulator and demodulator to reduce high-order terms to an extraordinarily low level, but also very stable lasers for energy modulation and demodulation to cancel each other out for facilitating beam transport. Reducing the repetition rate of reversible SSMB will highly increase the feasibility.

In this paper, we will show that it is possible to produce $10 \mathrm{kHz}$ coherent radiation without demodulation; therefore, the challenges will be greatly reduced. The energy-modulated beam needs to be damped to an equilibrium state by radiation damping, which limits the repetition rate. Nonetheless, numerical simulation results obtained for an extremely low emittance $3.5 \mathrm{GeV}$ storage ring adopting an advanced higher-order achromat (HOA) lattice demonstrate that the proposed angular dispersioninduced microbunching (ADM) scheme [20-23] generates coherent radiation near the soft $\mathrm{x}$-ray region and obtains a $10 \mathrm{kHz}$ repetition rate with an average brightness of $2.3 \times 10^{24}$ photons s${ }^{-1} \mathrm{~mm}^{-2} \mathrm{mrad}^{-2}(0.1 \% \text { bandwidth })^{-1}$, which is 2 orders of magnitude greater than fourth-generation synchrotron light sources. Accordingly, the results indicate that the proposed scheme will benefit synchrotron users in many respects.

The remainder of this paper is organized as follows. The ADM scheme is described in Sec. II, and its performance is demonstrated for a standard $3.5 \mathrm{GeV}$ electron beam based on numerical simulations. Section III presents the lattice design, linear optics, and nonlinear optics of the low emittance $3.5 \mathrm{GeV}$ storage ring, while the beam distortion, repetition rate, and brightness of the storage ring employing the ADM scheme are analyzed by numerical simulations in Sec. IV. Finally, discussions and conclusions are given in Sec. V.

\section{ANGULAR DISPERSION-INDUCED MICROBUNCHING SCHEME}

The ADM scheme [20-23] is a suitable method to produce high harmonics through a very weak modulation amplitude. This is because an electron beam gets very small vertical emittance, which is naturally preserved in an electron storage ring. We recall this scheme in Ref. [20], and the layout of it is shown in Fig. 1. A magnetic dipole $(B)$ is added upstream of the modulator $(M)$ (a seed laser propagates with an electron beam coaxially in an undulator modulating the electron beam energy at the wavelength of the laser), following a dogleg that consists of two dipoles with opposite polarity as the dispersion section $(D)$. The first dipole is used to introduce an angular dispersion into the electron beam, and then the seed laser pulse at an optical wavelength is employed to interact with the electron beam in the modulator to introduce a small energy modulation. After that, the energy modulation is converted into density modulation by the dogleg. The dispersive properties of the first dipole and the dogleg allow, if the parameters are chosen properly, the full compensation of the initial beam 


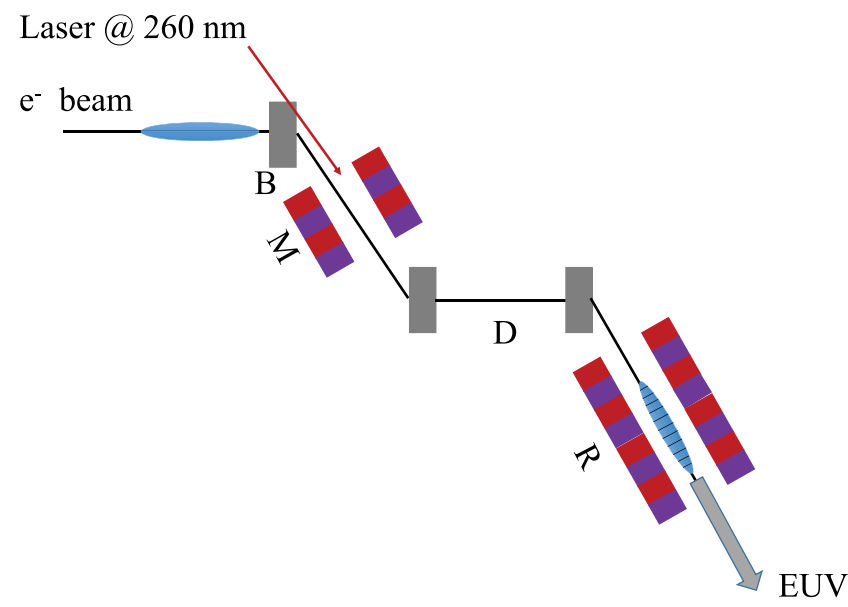

FIG. 1. Layout of the ADM scheme.

energy spread to produce very sharp microbunches at the EUV wavelength. A subsequent undulator, named the radiator $(R)$, is tuned so that intense, coherent radiation at a wavelength corresponding to one of these harmonics is emitted. This kind of electron beam would help to initiate intense coherent radiation at an EUV wavelength in the radiator. This scheme can be incorporated into a long straight section of the ring. The transverse dispersion generated by the dogleg can be fully compensated by another reversed dogleg after the radiator. This technique can make full use of the low emittance of the electron beams of a storage ring while effectively mitigating the detrimental effects from the large energy spread in the meantime.

Three-dimensional (3D) numerical simulations are employed here to demonstrate the possible performance of the ADM scheme, although a detailed matrix derivation and bunching factor analysis can be obtained elsewhere [21]. The electron beam is tracked through the beam line using the ELEGANT code with second-order transport effects taken into account [24]. The high-harmonic bunching factor is determined by the product of the dispersion generated in $D$ and the initial vertical divergence of the
TABLE I. Main parameters employed in the three-dimensional (3D) simulations applied for demonstrating the performance of the ADM scheme.

\begin{tabular}{lc}
\hline \hline Parameter & Value \\
\hline Energy [GeV] & 3.5 \\
Energy spread [MeV] & 3.6 \\
Emittance $(H, V)[\mathrm{pm} \mathrm{rad}]$ & $(25,25)$ \\
Laser wavelength [nm] & 260 \\
Laser pulse length [mm] & 5.4 \\
Laser power [MW] & 200 \\
Length of modulator $M$ [cm] & $1.2 \times 10$ \\
Peak magnetic field of $M[\mathrm{~T}]$ & 1.8 \\
Length of radiator $R$ [cm] & $0.6 \times 50$ \\
Peak magnetic field of $R[\mathrm{~T}]$ & 2.0 \\
Beta function at $B(H, V)[\mathrm{m}]$ & $(10,28)$ \\
Alpha function at $B(H, V)[\mathrm{m}]$ & $(0.5,0)$ \\
Length of dipole magnets [m] & 0.3 \\
Bending angle of $B$ [mrad] & 11.8 \\
Bending angle of the dipoles in $D$ [mrad] & 14.8 \\
Distance between two dipoles in $D$ [m] & 0.35 \\
Total length [m] & 5.45 \\
\hline \hline
\end{tabular}

electron beam. In addition, one can enhance the bunching factor for a given energy modulation amplitude by increasing the beta function at the entrance of the first dipole to reduce the vertical beam divergence or by increasing the strength of the first dipole to reduce the required dispersion provided by the dogleg. The beam parameters used in the 3D simulations are listed in Table I, where $H$ and $V$ denote the horizontal and vertical directions, respectively.

The longitudinal phase space of the electron beam was simulated through the ADM path length. The first dipole creates the correlation between the angular divergence and energy deviation of the electron beam. As the initial angular divergence of the electron beam is very small, the angular divergence represents the electron energy deviation after passing through the first dipole. The initial longitudinal phase space is shown in Fig. 2(a). After passing through the first dipole with a length of $0.3 \mathrm{~m}$ and a bending angle of about $11.8 \mathrm{mrad}$, the electron beam is sent into a short
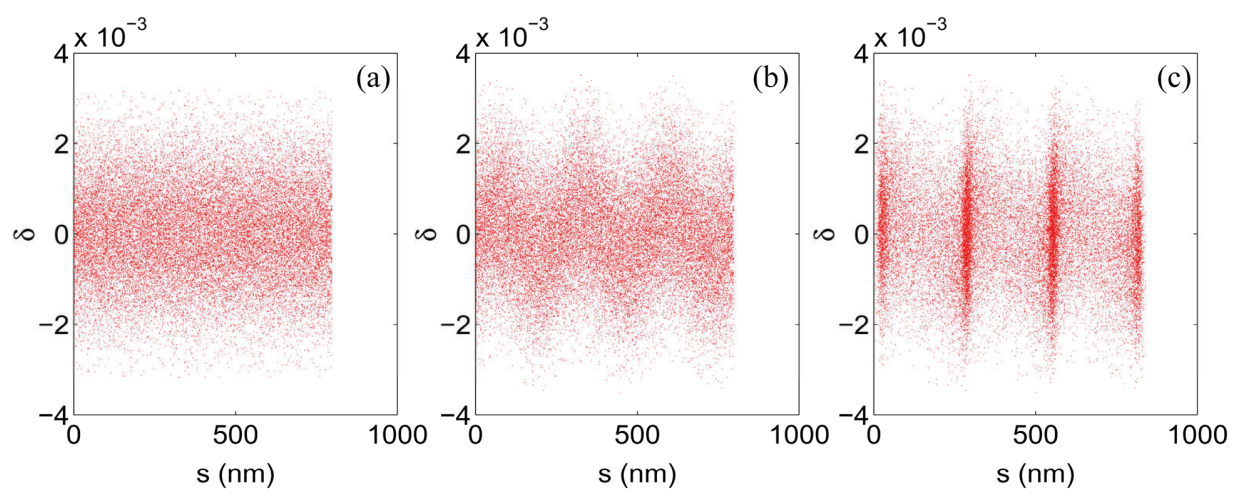

FIG. 2. Simulated longitudinal phase-space evolution of the electron beam: (a) initial phase space; (b) phase space at the exit of modulator $M$; (c) phase space at the entrance of radiator $R$. 


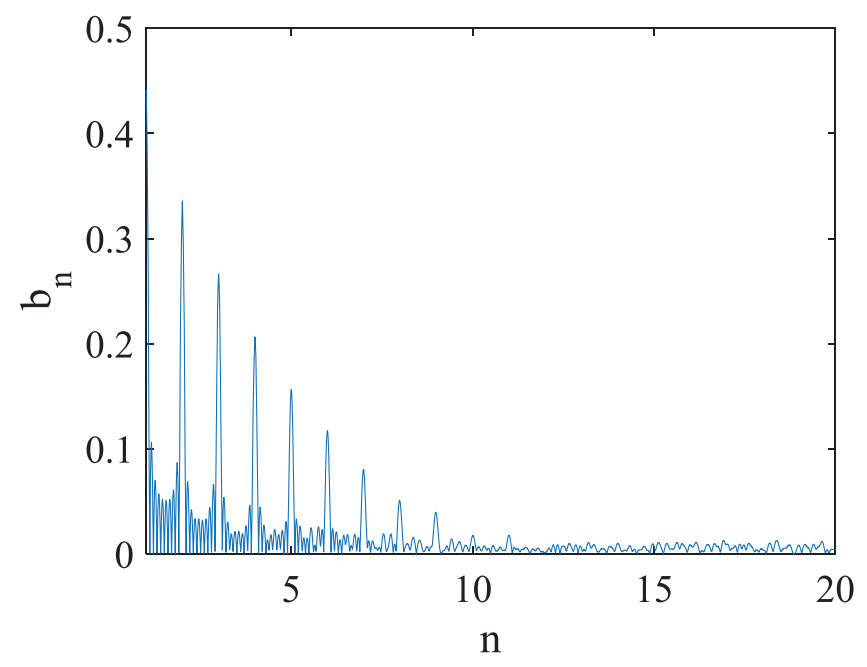

FIG. 3. Simulated bunching factors $b_{n}$ obtained by the ADM scheme as a function of the harmonic number $n$ with an energy modulation amplitude assigned as about 0.34 times that of the initial electron beam energy spread.

modulator to interact with the seed laser at wavelength $260 \mathrm{~nm}$, and then the phase space becomes Fig. 2(b). This energy modulation is then transformed into an associated density modulation by a dogleg, shown in Fig. 2(c). The dipole magnets in the dispersion section have a length of $0.3 \mathrm{~m}$ and a bending angle of about $14.8 \mathrm{mrad}$. The distance between the two dipoles in the dispersion section is $0.35 \mathrm{~m}$.

We also calculated the bunching factors $b_{n}$ obtained by the ADM scheme based on the numerical simulations as a function of the harmonic number $n$ with an energy modulation amplitude assigned as about 0.34 times that of the initial electron beam energy spread. The results of analysis are presented in Fig. 3. One can find that $b_{n}$ is about 0.12 at $n=6$, which represents coherent radiation at a wavelength of $44.3 \mathrm{~nm}$. The bunching factor is sufficiently large to effectively suppress the electron beam shot noise and generate coherent radiation in the radiator.

\section{STORAGE RING DESIGN}

The storage ring light sources currently running in the world are mostly third-generation light sources. ADM can be adopted in the third-generation light sources, yet, for advanced radiation production, ADM based on the fourthgeneration light source is more attractive. ADM adopted in the fourth-generation light source will increase the average brightness by 2 orders of magnitude. Furthermore, studying ADM in the fourth-generation light source will benefit the future investigation of reversible SSMB, because an accurate reverse modulation needs an ultralow emittance to reduce the high-order effects. ADM requires an ultralow vertical emittance to produce high harmonics; in the thirdgeneration light source, transverse coupling can be controlled to a very high degree [25], but, for long-term operation, a $0.1 \%$ change in transverse coupling has a great impact on the vertical emittance, which will affect the bunching factor. While the horizontal emittance of the fourth-generation light source is already extremely low, the vertical emittance is not sensitive to the transverse coupling. As such, the application of ADM to fourth-generation synchrotron light sources is more attractive for advanced radiation production.

The fourth generation of light sources has been emerging based on the multibend achromat (MBA) lattice types $[26,27]$. The MBA lattice types exploit the inverse cubic dependence of emittance on the number of bending magnets. By choosing a very small bending angle per dipole, the emittance can be dramatically reduced. The synchrotron radiation source MAX IV in Lund, Sweden, is the first light source that was successfully commissioned with this new lattice type $[28,29]$. The hybrid multibend achromat (HMBA) is another effective way to decrease emittance. It was employed for the first time in the upgrade plan of European Synchrotron Radiation Facility [30]. In the hybrid lattice cell, the sextupoles are located in the two dispersion bumps at both sides of the cell, with sextupoles of each family separated by a - I transformation (the phase advance between the sextupoles at both ends of the cell is set to $3 \pi$ in the horizontal plane and $\pi$ in the vertical plane, and $\mathbf{I}$ is the unity matrix) to cancel part of their nonlinear effects. Many other light sources worldwide follow this idea, such as Advanced Photon Source Upgrade (APS-U) [31], High Energy Photon Source (HEPS) [32], and Hefei Advanced Light Facility (HALF) [33,34].

As an alternative, a scheme known as HOA develops an MBA lattice where chromaticity-correcting sextupole magnets are distributed in each unit cell with strict phase advances over the cell such as to cancel basic geometric and chromatic resonance driving terms [35]. One important addition is the use of longitudinal-gradient bends (LGBs) to suppress the dispersion at the LGB center, where the field is strongest $[36,37]$. Furthermore, the focusing quadrupoles can be offset radially in order to provide aggressive reverse bending (RB) [38], which allows tuning the dispersion independently from the beta functions and, thereby, optimizes the unit cell for ultralow emittance and appropriate phase advance. Swiss Light Source-2 is a typical example of using this scheme [39].

The optical $\beta$ functions in the horizontal and vertical directions and the horizontal dispersion $\eta_{x}$ of the unit cell in our HOA lattice design are presented in Fig. 4(a). The cell has a net bending angle of $(10 / 6)^{\circ}$, which is composed of the $1.95^{\circ}$ deflection of the center LGB (yellow rectangle) and the $2 \times\left(-0.14^{\circ}\right)$ deflection of the two RBs (orange rectangles). In addition, the magnetic field strength $K$ of the quadrupole magnets and the magnetic field profile $B$ of the LGB are presented in Fig. 4(b). We note from Fig. 4(b) that the two regions lying outside the range of the LGB have very small deflection angles and defocusing quadrupole 

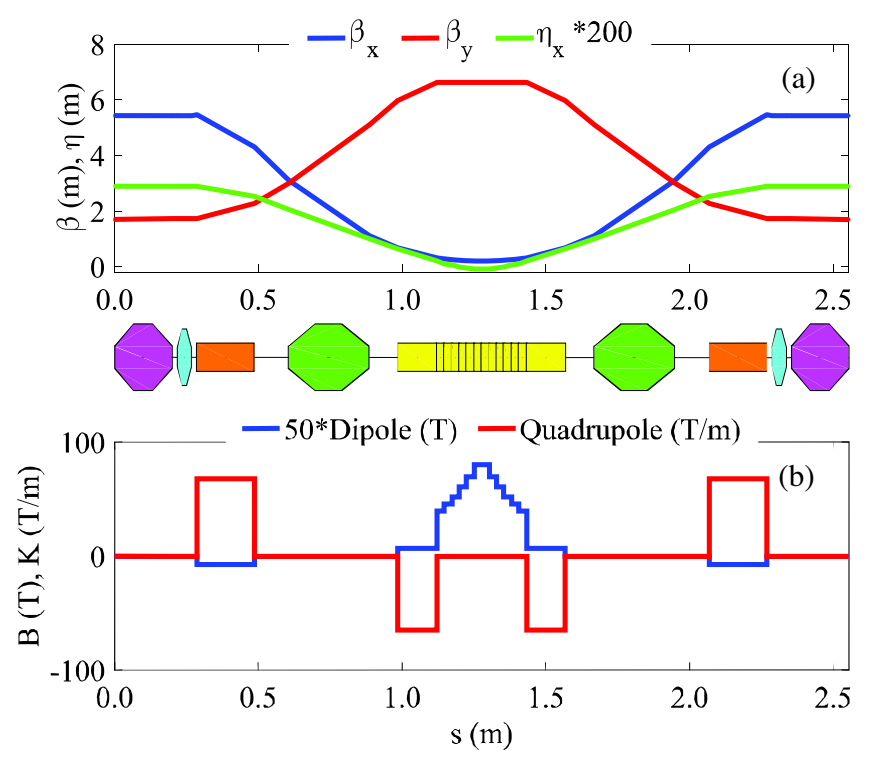

FIG. 4. (a) Optical functions of the unit cell in the proposed HOA lattice design, where LGB is given by the yellow rectangle, $\mathrm{RBs}$ are given by the orange rectangles, the sextupole magnets are given by the green and magenta hexagons, and the octupole magnets are given by the cyan hexagons. (b) Magnetic field strength $K$ of the quadrupole magnets and the magnetic field profile $B$ of the LGB for the unit cell.

fields. In order to achieve a higher-order achromat across the seven cells, the phase advances for the unit cell are then $(3 / 7,1 / 7) \times 2 \pi$ [40]. In addition, the ratio of the RB deflection angle to the total net deflection angle greatly affects the horizontal emittance $\varepsilon_{x}$ and momentum compression factor $\alpha_{c}$, as shown in Fig. 5 for the unit cell, where the RB deflection angle ratio is given by the color bar. Our design goal is to obtain an emittance value of about $20 \mathrm{pm}$ rad. Accordingly, we select a deflection angle of $-0.14^{\circ}$, which yields $\alpha_{c}=-2.4 \times 10^{-5}$ in the unit cell.

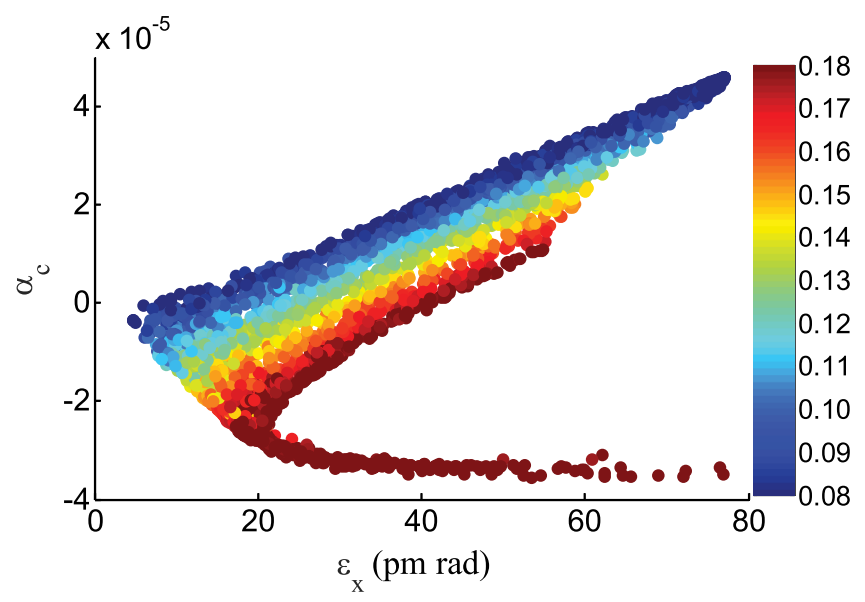

FIG. 5. Momentum compaction factor $\alpha_{c}$ versus the horizontal emittance $\varepsilon_{x}$ for RBs with different ratios of the RB deflection angle to the total net deflection angle denoted by the color bar.
The storage ring lattice consists of 36 seven-bend achromats separated by $4.7 \mathrm{~m}$ straight sections for insertion devices (IDs). Each of the achromats consists of five unit cells and two matching cells. The unit cells have a $(10 / 6)^{\circ}$ bending magnet, while the matching cells at the ends of the achromat have a $(10 / 12)^{\circ}$ bending magnet. The optical functions of one 7BA cell are shown in Fig. 6. The maximum strength of the dipole and quadrupole magnets is $1.6 \mathrm{~T}$ and $81.6 \mathrm{~T} / \mathrm{m}$, respectively. The horizontal and vertical tunes of one 7BA cell are set to $\mu_{x}=19 / 6$ and $\mu_{y}=7 / 6$, respectively, for the nonlinear cancellation [41].

The ADM cell includes a module for generating microbunching in the middle of the cell and two matching modules at its head and tail. The total length of the ADM cell is about $30 \mathrm{~m}$. As discussed in Sec. II, specific Twiss parameters are required at the entrance of the first dipole to generate a large bunching factor. In order to cancel the vertical dispersion generated by the dogleg and return the vertical plane to the standard plane, we need to add two dipoles and one dogleg to the structure shown in Fig. 1. In addition, some quadrupole magnets are needed to match the Twiss parameters of this cell with the Twiss parameters at the midpoint of the straight section of the storage ring. The complete horizontal layout of the ADM cell is shown in Fig. 7(a), and the optical functions of the ADM cell are presented in Fig. 7(b).

The 36 achromats of the storage ring are connected by the ADM cell and an injection cell, which consists of six focusing quadrupole magnets and four defocusing quadrupole magnets employed for beam injection. The layout of the entire storage ring is illustrated in Fig. 8, along with the $\beta$-function values in the injection cell. We note that the

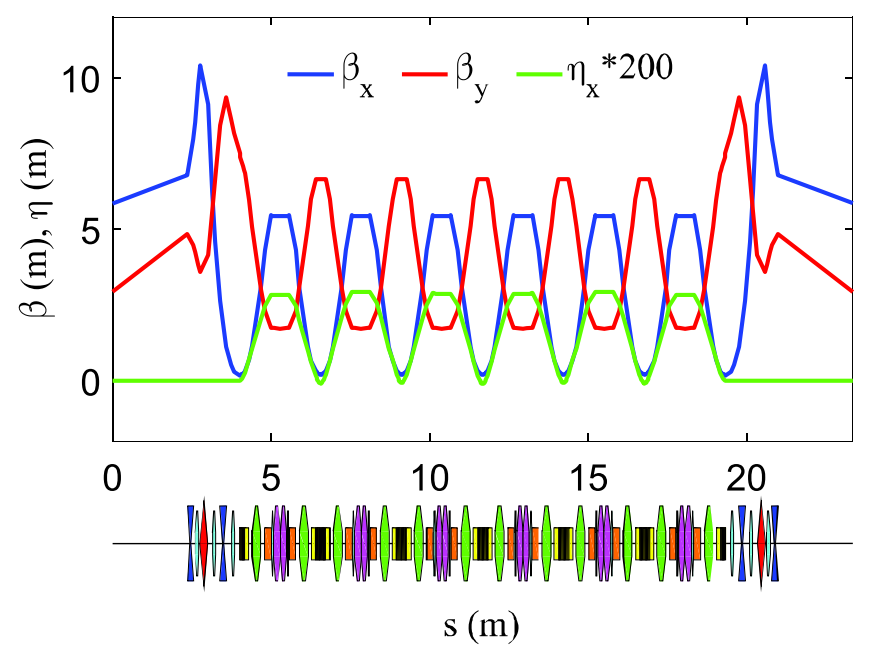

FIG. 6. Optical functions for one 7BA cell. Where the yellow elements are dipole magnets, the red and blue elements are focusing and defocusing quadrupole magnets, respectively, the green and magenta elements are focusing and defocusing sextupole magnets, respectively, and cyan elements are octupole magnets. 

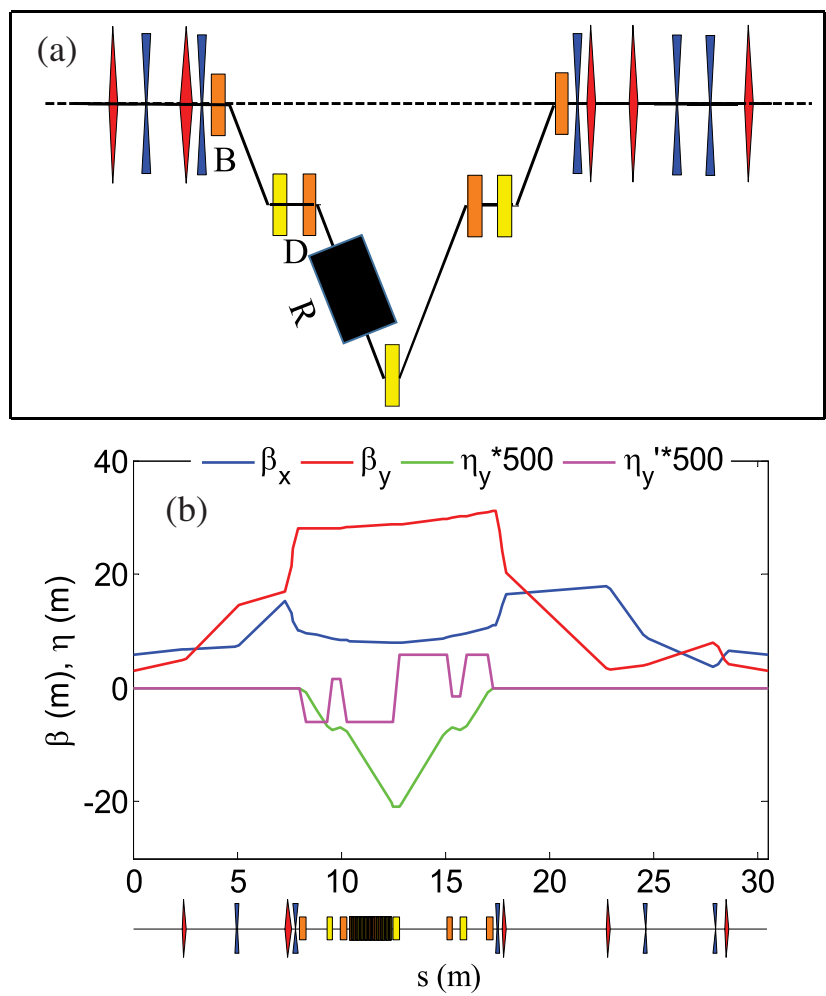

FIG. 7. (a) Complete horizontal layout of the ADM cell, where the blue and red elements represent defocusing quadrupole and focusing quadrupole magnets, respectively, the black rectangle represents the radiator $(R)$, the yellow rectangles represent normally polarized dipole magnets, and orange rectangles represent reverse polarized dipole magnets. (b) Optical functions for the ADM cell.

value of $\beta_{x}$ is about $18.5 \mathrm{~m}$ at the midpoint of the injection cell. The main parameters of the entire storage ring are listed in Table II. The dipole magnets in the ADM cell deflect in the vertical direction, and the undulator is located at a point where the vertical dispersion is relatively large. Therefore, both of these factors contribute to the vertical emittance. We note that the emittance after considering the intrabeam scattering (IBS) effect is $25 \mathrm{pm}$ rad in both the horizontal and vertical directions, as shown in Table II. However, the vertical emittance of the entire storage ring was determined to be $20 \mathrm{pm}$ rad using the beam-envelope matrix method [42].

The optimization of nonlinear optics is an enormous challenge for an ultralow emittance lattice. This is required to provide sufficient dynamic acceptance for injection, while strong nonlinearities are introduced by the sextupole magnets needed for correcting the natural chromaticity. Moreover, as can be seen from Fig. 8, the entire storage ring has only twofold symmetry, which brings additional challenges to the process.

The nonlinear design strategy is to realize the MBA arc as a higher-order achromat starting from the unit cell with two chromatic sextupole families. The cell tunes are chosen

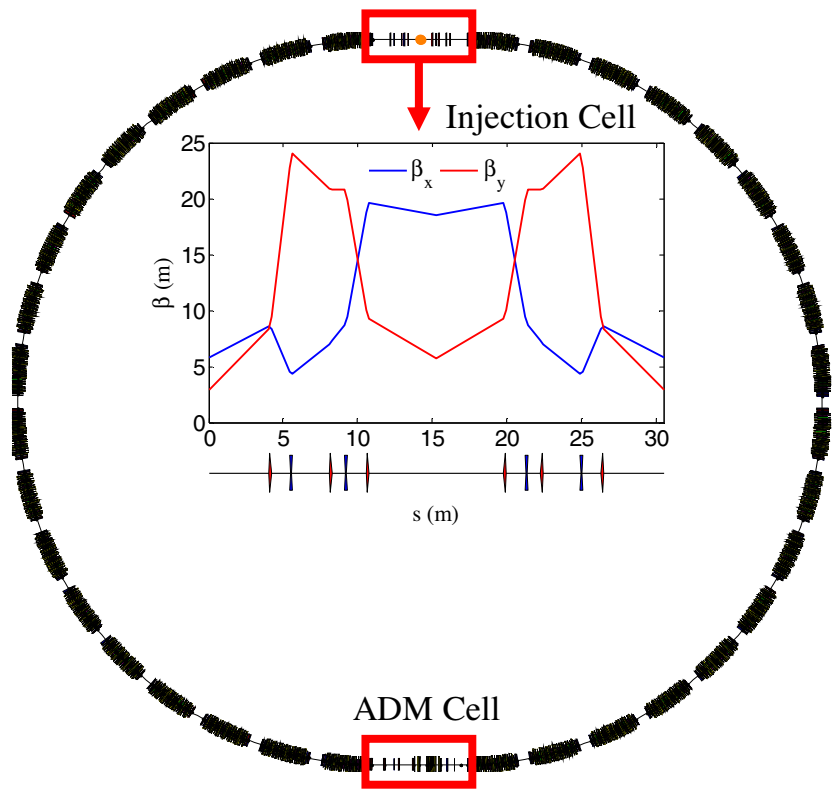

FIG. 8. Layout of the entire storage ring along with the $\beta$-function values in the injection cell.

so that all resonances up to the second order in the sextupole magnetic field strength cancel over the five unit cells and the two matching cells [41]. Then, the tune footprint is tailored to fit between major resonances using small octupole magnets, where three families of dispersionfree octupole magnets in the matching section mainly control the three amplitude-dependent tune shifts (ADTSs), while two families of dispersive octupoles in the arc mainly control the two second-order chromaticities. The sextupole magnets in the unit cells and match cells are in different families. Therefore, there are nine optimization knobs, which are four families of sextupole magnets and five families of octupole magnets. The nonlinear effects of

TABLE II. Main parameters of the entire storage ring.

\begin{tabular}{lc}
\hline \hline Parameter & Value \\
\hline Energy $[\mathrm{GeV}]$ & 3.5 \\
Circumference $[\mathrm{m}]$ & 900 \\
Emittance $(H, V)[\mathrm{pm} \mathrm{rad}]$ & $(20,20)$ \\
Emittance $(H, V)$ with IBS [pm rad] & $(25,25)$ \\
Betatron tune $(H, V)$ & $(115.16,43.27)$ \\
Energy spread & $1.05 \times 10^{-3}$ \\
Momentum compaction factor & $-1.5 \times 10^{-5}$ \\
Natural chromaticity $(H, V)$ & $(-258,-116)$ \\
Corrected chromaticity $(H, V)$ & $(-0.1,-0.1)$ \\
Damping time $(x, y, z)[\mathrm{ms}]$ & $(11.0,13.7,7.8)$ \\
Energy loss per turn $[\mathrm{MeV}]$ & 1.5 \\
rms bunch length $[\mathrm{mm}]$ & 1.8 \\
rf voltage $[\mathrm{MV}]$ & 2.15 \\
Length of straight section $[\mathrm{m}]$ & $4.7 \times 34+30 \times 2$ \\
Beta function in straight section $(H, V)[\mathrm{m}]$ & $(5.8,2.9)$ \\
\hline \hline
\end{tabular}



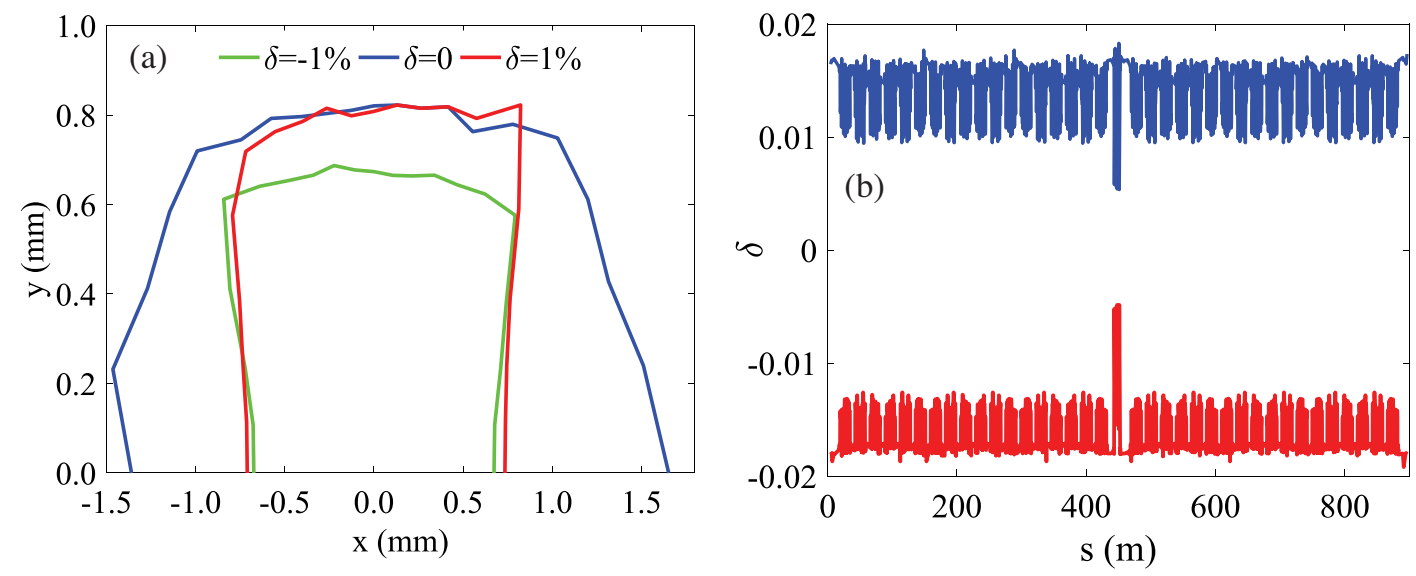

FIG. 9. (a) Dynamic aperture (DA) at the center of the injection section. (b) Local momentum apertures (MAs) for the entire storage ring.

the optics are reduced by optimizing the resonance drive terms (RDTs) [43] using multiobjective particle swarm optimization [44]. Two second-order chromaticity terms and three ADTSs are optimized while maintaining a corrected chromaticity value of -0.1 . Here, we note that a negative chromaticity does not induce head-tail instability when the momentum compression factor of the storage ring is less than zero.

After optimizing chromatic and resonance driving terms, we evaluate the dynamic aperture (DA) and local momentum apertures (MAs) of the designed lattice. The DA and local MAs were calculated using the ELEGANT code, and the particles were tracked for 2000 turns. The results of sixdimensional (6D) DA tracking at the center of the injection section are presented in Fig. 9(a) for different energy deviations $\delta$. The off-momentum DA is greatly reduced, and the optimization of the off-momentum DA of this lattice is very challenging. In addition, the local MAs for the entire storage ring are presented in Fig. 9(b). The local MAs are limited by the vertical dispersion bump in the ADM cell, where a third-harmonic cavity is used to increase the bunch length and, thereby, increase the beam lifetime. With a beam current of $150 \mathrm{~mA}$, the Touschek lifetime is $0.4 \mathrm{~h}$. In addition, the elastic scattering lifetime is $11.0 \mathrm{~h}$, and the inelastic scattering lifetime is $133.6 \mathrm{~h}$, which were calculated using the ELEGANT code (under the conditions: vacuum pressure $1 \mathrm{nTorr}$ and gas type $\mathrm{H}_{2}$ $65 \%, \mathrm{H}_{2} \mathrm{O} 2 \%, \mathrm{CH}_{4} 7 \%, \mathrm{CO} 22 \%$, and $\mathrm{CO}_{2} 4 \%$ ). Finally, the on-axis swap out scheme was employed for injection owing to the small DA.

\section{BEAM DISTORTION AND REPETITION RATE}

\section{A. Nonlinear optimization and beam distortion}

The octupole magnets can effectively adjust the secondorder chromaticities and ADTSs. This is illustrated in Table III by the two second-order chromaticities and three
ADTSs obtained before and after the optimization of the octupole magnets.

From Eq. (5) in Ref. [21], we obtain the energymodulated transport matrix as follows:

$$
\begin{aligned}
M 1 & =R_{D} \cdot R_{M} \cdot R_{B} \\
& =\left[\begin{array}{cccc}
1+h b \eta & L & h \eta & \eta-L b \\
0 & 1 & 0 & -b \\
b\left(1+h \xi_{D}\right) & \eta & 1+h \xi_{D} & \xi-\eta b \\
h b & 0 & h & 1
\end{array}\right],
\end{aligned}
$$

which is a product of the transport matrices $R_{B}, R_{M}$, and $R_{D}$, respectively, associated with the first dipole $B$, modulator $M$, and dispersion section $D$. Here, $h=k_{s} \Delta \gamma / \gamma$, where $k_{s}$ is the wave number of the seed laser, $\Delta \gamma$ is the energy modulation amplitude induced by the seed laser, $\gamma$ is the relativistic parameter for the mean beam energy, $b$ is the bending angle of the first dipole $B, \eta$ is the dispersion generated in dispersion section $D, L=L_{M}+L_{D}$, where $L_{M}$ is the length of modulator $M$ and $L_{D}$ is the length of dispersion section $D$, and $\xi=\xi_{M}+\xi_{D}$, where $\xi_{M}$ is the momentum compaction generated in the modulator $M$ and $\xi_{D}$ is the momentum compaction generated in dispersion section $D$. Without energy modulation, the transport matrix is simplified as follows:

TABLE III. RDTs before and after the optimization of octupole magnets.

\begin{tabular}{lcc}
\hline \hline Parameter & Before optimization & After optimization \\
\hline h11002 & -483 & -135 \\
h00112 & 350 & 307 \\
h22000 & $1.77 \times 10^{5}$ & $4.66 \times 10^{5}$ \\
h11110 & $2.18 \times 10^{6}$ & $7.88 \times 10^{4}$ \\
h00220 & $4.53 \times 10^{6}$ & $1.84 \times 10^{4}$ \\
\hline \hline
\end{tabular}



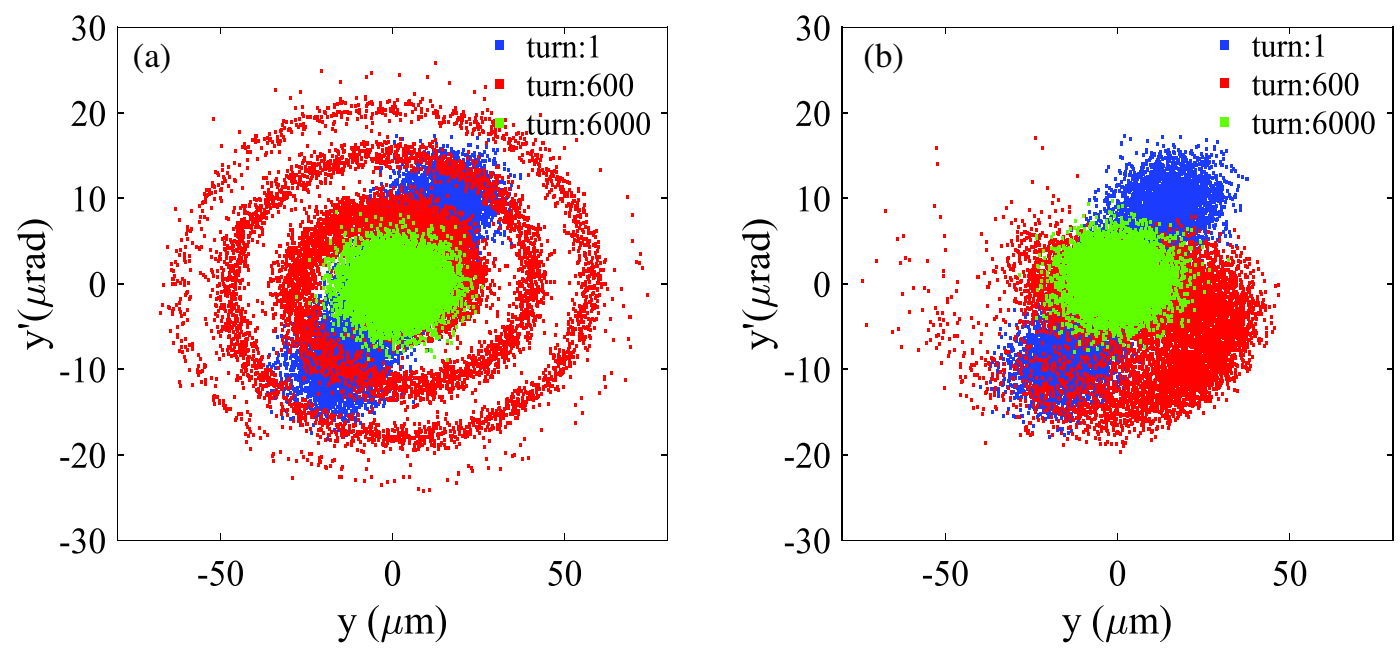

FIG. 10. Simulated beam distribution in the vertical plane after energy modulation for different turn numbers: (a) before octupole magnet optimization and (b) after octupole magnet optimization.

$$
M 2=R_{D} \cdot R_{B}=\left[\begin{array}{cccc}
1 & L_{D} & 0 & \eta-L_{D} b \\
0 & 1 & 0 & -b \\
b & \eta & 1 & \xi_{D}-\eta b \\
0 & 0 & 0 & 1
\end{array}\right]
$$

Comparing $M 1$ and $M 2$, one notes that $R_{11}$ of $M 1$ gets an additional term $h b \eta$. This term is related to the energy modulation, which distorts the transverse phase space when the electron beam interacts with the laser in the undulator.

We investigated the effect of nonlinear optimization on beam distortion by means of simulations using 10000 particles and ELEGANT code. The simulated beam distributions in the vertical plane after a single energy modulation for different turn numbers before [case (a)] and after [case (b)] octupole magnets optimization are shown in Figs. 10(a) and 10(b), respectively. The initial beam is a Gaussian distribution at the modulation point which is in the position of the modulator. Turn 1 represents the beam phase space distribution at the first turn after energy modulation. The red and green dots represent the natural damping beam distribution at the 600th and 6000th turns, respectively, after one energy modulation. The next modulation will act after the beam has damped to an equilibrium state. The area of the phase space occupied by the particles represents the emittance. However, despite the fact that the emittance is postulated according to Liouville's theorem to be a constant after the beam is modulated, we note that the vertical phase spaces of the electrons at the 600th turn shown in Fig. 10 differ before and after octupole magnet optimization. This is because the nonlinearity of the storage ring increasingly distorts the phase space through filamentation over many turns, which leads to the false image of the increasing of the emittance when calculating the vertical emittance $\varepsilon_{y}$ according to the statistical definition $\varepsilon_{y}=\sqrt{\left\langle y^{2}\right\rangle\left\langle y^{\prime 2}\right\rangle-\left\langle y \cdot y^{\prime}\right\rangle^{2}}$, where $y$ is the vertical coordinate and $y^{\prime}$ is the vertical divergence. This emittance is denoted as the root mean square (rms) projected emittance [45]. Accordingly, the octupole magnets are employed to limit this rms projected emittance, because the light source station receives far-field radiation and the brightness will decrease with increasing $\varepsilon_{y}$.

This issue is analyzed further by plotting the value of $\varepsilon_{y}$ obtained with respect to the number of turns after a single energy modulation in Fig. 11 both before and after octupole magnet optimization. We note from Fig. 11 case (a) that $\varepsilon_{y}$ increased very quickly without octupole magnets optimization and attained a maximum value of about $285 \mathrm{pm}$ rad at the 150th turn. At this time, the vertical phase space was very much more diffuse than that shown in Fig. 10(a).

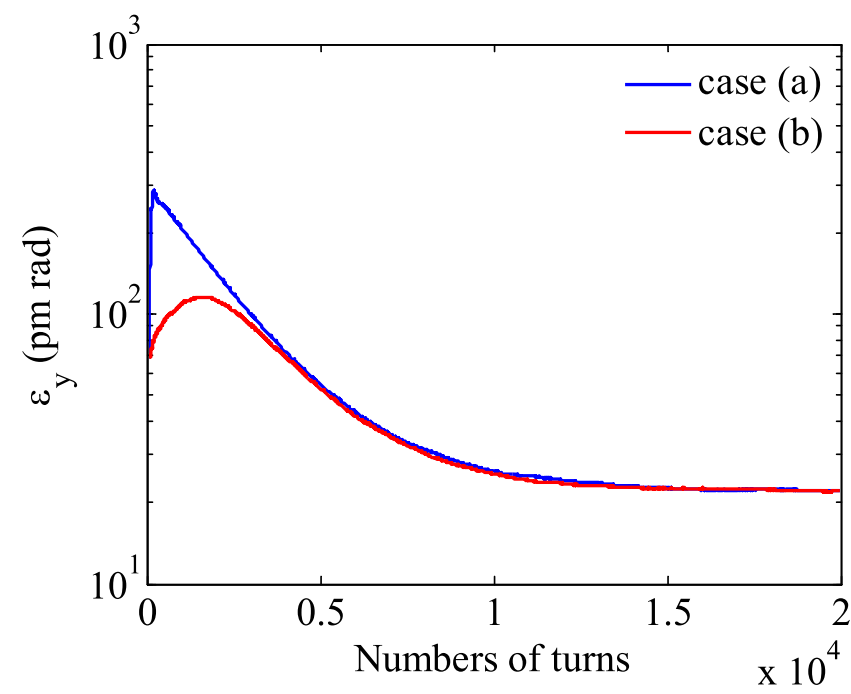

FIG. 11. Simulated vertical emittance $\varepsilon_{y}$ obtained after a single energy modulation with respect to the number of turns before octupole magnet optimization [case (a)] and after octupole magnet optimization [case (b)]. 
At the 6000th turn, $\varepsilon_{y}$ was damped to about $43 \mathrm{pm} \mathrm{rad}$. Accordingly, the damping time $\left(\tau_{a 1}\right)$ of the first 6000 turns was about $8.8 \mathrm{~ms}$. During this interval of turns, the phase space distortion was due to a high degree of nonlinearity, and the energy modulation was transferred to the vertical direction. At this point, $\tau_{a 1}$ is approximately the longitudinal damping time. With subsequent turns, the damping time progressively decreased. At the 11000 th turn, $\varepsilon_{y}$ was damped to an equilibrium value of about $25 \mathrm{pm} \mathrm{rad}$, and the damping time $\left(\tau_{a 2}\right)$ between the 6000th and 11 000th turns was about $23.4 \mathrm{~ms}$. In contrast, $\varepsilon_{y}$ increased much more slowly after octupole magnet optimization, and $\varepsilon_{y}$ attained a maximum value of about $115 \mathrm{pm}$ rad at the 1800th turn, while $\varepsilon_{y}$ was damped to about $42 \mathrm{pm}$ rad at the 6000th turn. Accordingly, the damping time of the first 6000 turns $\left(\tau_{b 1}\right)$ was about $11.6 \mathrm{~ms}$, where $\tau_{b 1}$ is approximately the vertical damping time. Finally, $\varepsilon_{y}$ was damped to an equilibrium state of approximately $25 \mathrm{pm}$ rad at the 10000 th turn. The damping time $\left(\tau_{b 2}\right)$ between the 6000th and 10000 th turns was about $19.5 \mathrm{~ms}$.

These results can be further analyzed to estimate the obtainable repetition rate. Here, the entire ring circumference is about $900 \mathrm{~m}$, which, when using a radio frequency (rf) of $500 \mathrm{MHz}$, represents 1500 buckets, among which 300 buckets are each filled with a charge of $1.5 \mathrm{nC}$. Therefore, modulating the electron bunches in turn to produce coherent radiation according to this filling pattern with a total average current of $150 \mathrm{~mA}$ yields a repetition rate of $10 \mathrm{kHz}$.

\section{B. Brightness and bandwidth}

The possible performance of the proposed ADM scheme employing a 3-m-long helical undulator with a period of $6 \mathrm{~cm}$ was evaluated according to 3D simulations for sixthharmonic $(44.3 \mathrm{~nm})$ radiation, which were conducted using the GENESIS code [46] along with the bunched electron beam simulated using the ELEGANT code based on the optimized storage ring. It is worth noting that this photon energy is appropriate for various important applications, such as superhigh-energy resolution angle-resolved photoemission spectroscopy [47]. The simulation results obtained for the output power and single-shot spectrum of the radiation emitted by the radiator are presented in Figs. 12(a) and 12(b), respectively. One can find that the peak power is greater than $1 \mathrm{MW}$ (i.e., $\sim 10^{12}$ photons per pulse). In addition, the spectral bandwidth in terms of the full width at half maximum (FWHM) is only $0.3 \mathrm{meV}$, which is quite close to the Fourier transform limit. Taking these results together with a repetition rate of $10 \mathrm{kHz}$ yields a spectral brightness of about $2.3 \times$ $10^{24}$ photons $^{-1} \mathrm{~mm}^{-2} \mathrm{mrad}^{-2}(0.1 \% \text { bandwidth })^{-1}$, which is more than 2 orders of magnitude greater than the spontaneous emission obtained with the same electron beam and undulator in a general fourth-generation synchrotron light
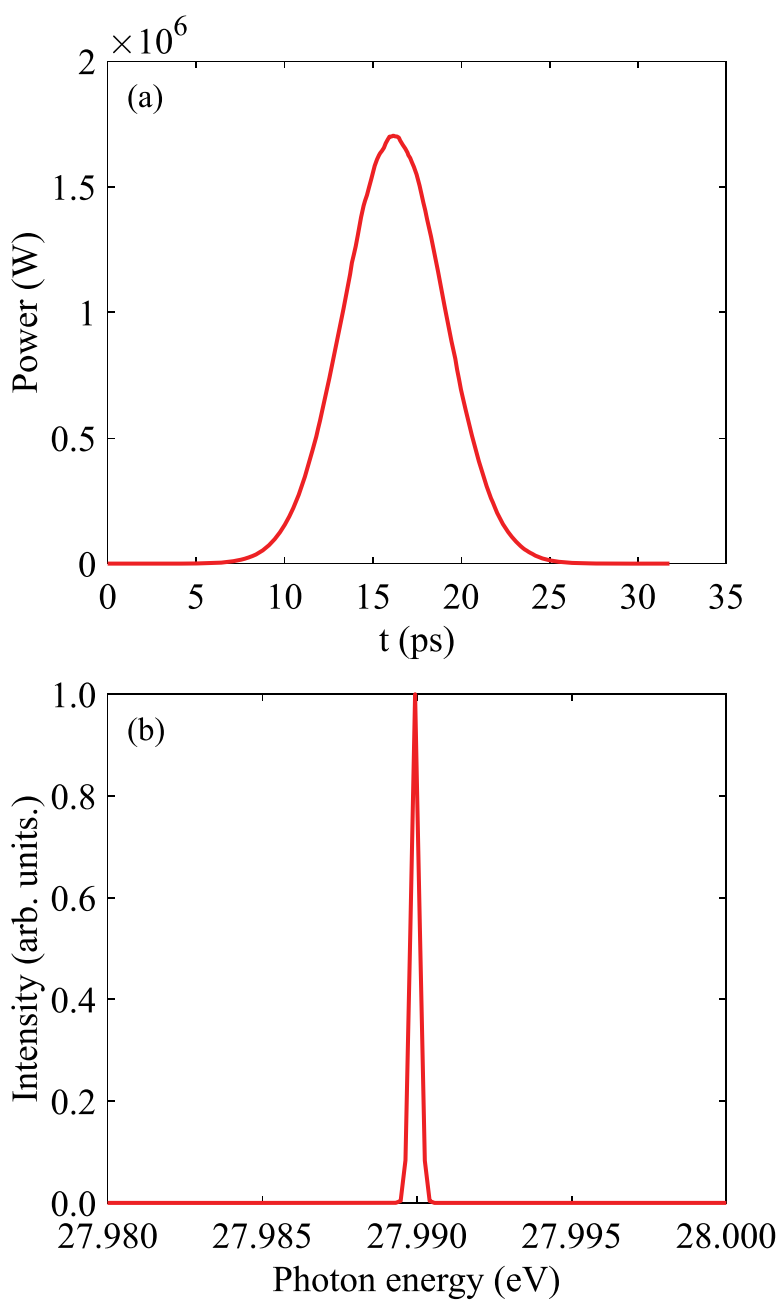

FIG. 12. Primary characteristics of the radiation pulse emitted by the radiator of the ADM cell: (a) output power; (b) single-shot spectrum.

source. In addition, the optical flux attains a value of $10^{16}$ photons/s. Finally, the extremely narrow band of the produced radiation eliminates the need for a monochromator in the beam line, which allows for the full usage of the available optical flux.

\section{DISCUSSIONS AND CONCLUSIONS}

It is worth pointing out that the operation of the proposed electron storage ring at $10 \mathrm{kHz}$ in this scheme results in 300 bunches being modulated and equidistantly spread out over the first 10000 turns in the ring. Beam line stations dedicated to this scheme will therefore achieve synchrotron radiation with extremely high coherence, but the brightness seen by other users will be reduced. We can calculate from the optimized storage ring in Fig. 11 that the vertical emittance actually seen by other users is $62.5 \mathrm{pm} \mathrm{rad}$, and the brightness seen by other users will decrease by a factor of 2.5. However, the brightness for other users is also acceptable. 
The performance of this ADM scheme for generating coherent radiation near the soft $\mathrm{x}$-ray region without demodulation in conjunction with the optimized storage ring design with a circumference of $900 \mathrm{~m}$ was demonstrated by numerical simulations to obtain a repetition rate of $10 \mathrm{kHz}$, a spectral bandwidth of $0.3 \mathrm{meV}$, and a spectral brightness of $2.3 \times 10^{24}$ photons s $\mathrm{mm}^{-1} \mathrm{mrad}^{-2}$ $(0.1 \% \text { bandwidth })^{-1}$. The extremely narrow band of the produced radiation eliminates the need for a monochromator in the beam line, which allows for the full usage of the available optical flux. Moreover, the electron beam in the storage ring is highly stable, which ensures that the obtained radiation will also be very stable, as is required by users for enabling highly accurate analyses.

\section{ACKNOWLEDGMENTS}

The authors are deeply grateful to Alex Chao (Tsinghua University) and Weishi Wan (ShanghaiTech University) for their guidance during this study. We also thank Zhenghe Bai, Tong Zhang, and Penghui Yang at the University of Science and Technology of China (USTC) for their helpful discussions regarding the storage ring lattice design. This work is supported by the National Natural Science Foundation of China (No. 11975300) and the National Key Research and Development Program of China (No. 2016YFA0402001).

[1] S. Kumar, Next generation light sources and applications, arXiv:1807.11084.

[2] C. Feng and H. X. Deng, Review of fully coherent freeelectron lasers, Nucl. Sci. Tech. 29, 160 (2018).

[3] Z. Huang and K. J. Kim, Review of x-ray free-electron laser theory, Phys. Rev. ST Accel. Beams 10, 034801 (2007).

[4] C. Pellegrini, A. Marinelli, and S. Reiche, The physics of X-ray free-electron lasers, Rev. Mod. Phys. 88, 015006 (2016).

[5] Z. T. Zhao, Storage ring light sources, Rev. Accel. Sci. Techol. 03, 57 (2010).

[6] S. Di Mitri, One way only to synchrotron light sources upgrade?, J. Synchrotron Radiat. 25, 1323 (2018).

[7] Y. Cai, Y. Ding, R. Hettel, Z. Huang, L. Wang, and L. Xiao, An X-ray free electron laser driven by an ultimate storage ring, SLAC Report No. SLAC-PUB-15380, 2013.

[8] D. Xiang and W. Wan, Generating Ultrashort Coherent Soft X-Ray Radiation in Storage Rings Using AngularModulated Electron Beams, Phys. Rev. Lett. 104, 084803 (2010).

[9] G. Stupakov, Frequency multiplication using coherent radiation of a "snake" beam, Phys. Rev. Accel. Beams 16, 010702 (2013).

[10] S. Khan, K. Holldack, T. Kachel, R. Mitzner, and T. Quast, Femtosecond Undulator Radiation from Sliced Electron Bunches, Phys. Rev. Lett. 97, 074801 (2006).
[11] R. W. Schoenlein, S. Chattopadhyay, H. H. W. Chong, T. E. Glover, P. A. Heimann, C. V. Shank, A. A. Zholents, and M. S. Zolotorev, Generation of femtosecond pulses of synchrotron radiation, Science 287, 2237 (2000).

[12] C. Sun, C. Steier, and W. Wan, Vertical dispersion bump design for femto-second slicing beamline at the ALS, in Proceedings of the 3rd International Particle Accelerator Conference, IPAC2012, New Orleans, Louisiana, USA (JACoW, Geneva, 2012), pp. 1698-1700, http://accelconf .web.cern.ch/IPAC2012/papers/tuppp039.pdf.

[13] D. F. Ratner and A. W. Chao, Steady-State Microbunching in a Storage Ring for Generating Coherent Radiation, Phys. Rev. Lett. 105, 154801 (2010).

[14] N. Nakamura, Review of ERL projects at KEK and around the world, in Proceedings of the 3rd International Particle Accelerator Conference, IPAC2012, New Orleans, Louisiana, USA (JACoW, Geneva, 2012), pp. 1040-1044, http://accelconf.web.cern.ch/IPAC2012/papers/tuxb02 .pdf.

[15] A. Chao, X. Deng, W. Huang, T. Rui, C. Tang et al., A compact high-power radiation source based on steady-state microbunching mechanism, SLAC Report No. SLACPUB-17241, 2018.

[16] C. Tang, X. Deng, A. Chao, W. Huang, T. Rui, J. Feikes, J. Li, M. Ries, A. Hoehl, D. Ratner et al., An overview of the progress on SSMB, in Proceedings of the 60th ICFA Advanced Beam Dynamics Workshop on Future Light Sources, FLS2018, Shanghai, China, 2018 (JACoW, Geneva, 2018), pp. 166-170, http://accelconf.web.cern .ch/fls2018/papers/thp2wb02.pdf.

[17] Z. Pan, T. Rui, W. Wan, A. Chao, X. Deng, Y. Zhang, W. Huang, and C. Tang, A storage ring design for steady-state microbunching to generate coherent EUV light source, in Proceedings of the 39th International Free Electron Laser Conference, FEL2019, Hamburg, Germany, 2019 (JACoW, Geneva, 2019), pp. 700-703, https://accelconf.web .cern.ch/AccelConf/fel2019/papers/thp055.pdf.

[18] X. J. Deng, R. Klein, A. W. Chao, A. Hoehl, W. H. Huang, J. Li, J. Lubeck, Y. Petenev, M. Ries, I. Seiler, C. X. Tang, and J. Feikes, Widening and distortion of the particle energy distribution by chromaticity in quasi-isochronous rings, Phys. Rev. Accel. Beams 23, 044001 (2020).

[19] X. J. Deng, A. W. Chao, J. Feikes, W. H. Huang, M. Ries, and C. X. Tang, Single-particle dynamics of microbunching, Phys. Rev. Accel. Beams 23, 044002 (2020).

[20] C. Li, C. Feng, B. Jiang, and A. Chao, Lattice design for the reversible SSMB, Proceedings of the 10th International Particle Accelerator Conference, IPAC2019, Melbourne, Australia, 2019 (JACoW, Geneva, 2019), pp. 1507-1509, https://accelconf.web.cern.ch/ipac2019/ papers/tupgw045.pdf.

[21] C. Feng and Z. Zhao, A storage ring based free-electron laser for generating ultrashort coherent EUV and x-ray radiation, Sci. Rep. 7, 4724 (2017).

[22] X. Wang, C. Feng, T. Liu, Z. Zhang, C. Y. Tsai, J. Wu, C. Yang, and Z. Zhao, Angular dispersion enhanced prebunch for seeding ultrashort and coherent EUV and soft x-ray free-electron laser in storage rings, J. Synchrotron Radiat. 26, 677 (2019). 
[23] C. Feng, B. Jiang, C. Li, X. Wang, Z. Zhao, and A. W. Chao, Generating intense coherent EUV radiation via three-dimensional manipulation of the electron beam in storage rings, in Compact EUV \& X-Ray Light Sources (Optical Society of America, Washington, D.C., 2018), pp. EW4B-3.

[24] M. Borland, Elegant: A flexible SDDS-compliant code for accelerator simulation, Advanced Photon Source Report No. LS-287, 2000.

[25] R. Dowd, M. Boland, G. LeBlanc, and Y-R. E. Tan, Achievement of ultralow emittance coupling in the Australian Synchrotron storage ring, Phys. Rev. Accel. Beams 14, 012804 (2011).

[26] D. Einfeld, J. Schaper, and M. Plesko, Design of a diffraction limited light source (DIFL), in Proceedings of the Particle Accelerator Conference, Dallas, TX, 1995 (IEEE, New York, 1995), pp. 177-179.

[27] D. Einfeld, M. Plesko, and J. Schaper, First multi-bend achromat lattice consideration, J. Synchrotron Radiat. 21, 856 (2014).

[28] P. F. Tavares, S. C. Leemann, M. Sjöström, and Å. Andersson, The MAX IV storage ring project, J. Synchrotron Radiat. 21, 862 (2014).

[29] P. F. Tavares, E. A. Dmour, Å. Andersson, F. Cullinan et al., Commissioning and first-year operational results of the MAX IV $3 \mathrm{GeV}$ ring, J. Synchrotron Radiat. 25, 1291 (2018).

[30] L. Farvacque, N. Carmignani, J. Chavanne, A. Franchi, G. Le Bec, S. Liuzzo, B. Nash, T. Perron, and P. Raimondi, A low-emittance lattice for the ESRF, in Proceedings of the4th International Particle Accelerator Conference, IPAC2013, Shanghai, China (JACoW, Geneva, 2013), pp. 79-81, http://accelconf.web.cern.ch/IPAC2013/ papers/mopea008.pdf.

[31] M. Borland, V. Sajaev, Y. Sun, and A. Xiao, Hybrid sevenbend-achromat lattice for the Advanced Photon Source upgrade, in Proceedings of the 6th International Particle Accelerator Conference, IPAC2015, Richmond, VA, USA, 2015 (JACoW, Geneva, 2015), pp. 1776-1779, http:// accelconf.web.cern.ch/IPAC2015/papers/tupje063.pdf.

[32] Y. Jiao, G. Xu, X. H. Cui, Z. Duan, Y. Y. Guo, P. He, D. H. Ji et al., The HEPS project, J. Synchrotron Radiat. 25, 1611 (2018).

[33] Z. Bai, P. Yang, Z. Yang, W. Li, and L. Wang, Design of the second version of the HALS storage ring lattice, in Proceedings of the 9th International Particle Accelerator Conference, IPAC2018, Vancouver, BC, Canada, 2018 (JACoW, Geneva, 2018), pp. 4601-4604, http://accelconf .web.cern.ch/ipac2018/papers/thpmk121.pdf.
[34] P. Yang, Z. Bai, Z. Zhang, D. Xu, and L. Wang, Design of a hybrid ten-bend-achromat lattice for a diffraction-limited storage ring light source, Nucl. Instrum. Methods Phys. Res., Sect. A 943, 162506 (2019).

[35] J. Bengtsson and A. Streun, Robust design strategy for SLS-2, Report No. SLS2-BJ84-001-2, 2017.

[36] A. Streun and A. Wrulich, Compact low emittance light sources based on longitudinal gradient bending magnets, Nucl. Instrum. Methods Phys. Res., Sect. A 770, 98 (2015).

[37] B. Riemann and A. Streun, Low emittance lattice design from first principles: Reverse bending and longitudinal gradient bends, Phys. Rev. Accel. Beams 22, 021601 (2019).

[38] A. Streun, The anti-bend cell for ultralow emittance storage ring lattices, Nucl. Instrum. Methods Phys. Res., Sect. A 737, 148 (2014).

[39] A. Streun, T. Garvey, L. Rivkin, V. Schlott, T. Schmidt, P. Willmott, and A. Wrulich, SLS-2 - The upgrade of the Swiss Light Source, J. Synchrotron Radiat. 25, 631 (2018).

[40] A. Verdier, Resonance free lattices for A.G. machines, in Proceedings of the 18th Particle Accelerator Conference, New York, 1999 (IEEE, New York, 1999), pp. 398-400.

[41] Y. Cai, K. Bane, R. Hettel, Y. Nosochkov, and M. H. Wang, Ultimate storage ring based on fourth-order geometric achromats, Phys. Rev. Accel. Beams 15, 054002 (2012).

[42] K. Ohmi, K. Hirata, and K. Oide, From the beam-envelope matrix to synchrotron-radiation integrals, Phys. Rev. E 49, 751 (1994).

[43] J. Bengtsson, The sextupole scheme for the Swiss Light Source (SLS): An analytic approach, SLS Note 9/97, 1997.

[44] X. Huang and J. Safranek, Nonlinear dynamics optimization with particle swarm and genetic algorithms for SPEAR3 emittance upgrade, Nucl. Instrum. Methods Phys. Res., Sect. A 757, 48 (2014).

[45] A. Franchi, L. Farvacque, J. Chavanne, F. Ewald, B. Nash, K. Scheidt, and R. Tomás, Vertical emittance reduction and preservation in electron storage rings via resonance driving terms correction, Phys. Rev. Accel. Beams 14, 034002 (2011).

[46] S. Reiche, GENESIS 1.3: A fully 3D time-dependent FEL simulation code, Nucl. Instrum. Methods Phys. Res., Sect. A 429, 243 (1999).

[47] G. Liu, G. Wang, Y. Zhu, H. Zhang, G. Zhang et al., Development of a vacuum ultraviolet laser-based angleresolved photoemission system with a superhigh energy resolution better than $1 \mathrm{meV}$, Rev. Sci. Instrum. 79, 023105 (2008). 